\title{
The Effect of Messaging on School Attendance
}

\author{
Aditya Mittal \\ Henry M. Gunn High School, Palo Alto, CA 94306 \\ email: adi@mittal.net
}

\begin{abstract}
School absenteeism is a critical issue in many K-12 districts. There have been previous studies that have looked at the problem but they have focused on at-risk students in underachieving districts. This study looked at the same problem at the other end of the spectrum: why do some of the top students in high performance schools miss classes consistently? And can messaging be used to boost attendance rates? Our hypothesis is that a large fraction of the absences at the higher end of the performance curve are strategic absentees: those who cut a class as a need to prepare for another one. This subcategory of chronic absenteeism is the focus of this paper. This subcategory differs in their academic motivation and attendance patterns from those studied earlier. We examined four different communication models: direct and indirect communication using both positive and negative information. The results, though preliminary, were unexpected: (i) none improved attendance, (ii) most seemed to marginally worsen it, contrary to previous results, and (iii) likely due to reactance.
\end{abstract}

Keywords: messaging, school absenteeism, reactance

\section{Introduction}

School absenteeism is a critical problem for many reasons ranging from state funding in public schools to student outcomes and their implications for future opportunities. The US Dept of Education found that absenteeism is a better predictor of student drop out rates than test scores. While there have been numerous studies that have looked at the problem, most of those studies focused on problematic districts that had high rates of chronic absenteeism amongst at-risk students. In contrast, our work examined the issue at a top decile school district, with high achieving students, and specifically looked at the question whether the causes of chronic absenteeism might be different. We looked at students in one of the two high schools in the district: with an enrollment of over 2,000 students, over $99 \%$ of whom graduate on time and go to college, absenteeism should not have been a problem. However, on any given day, at least 5\% of students are absent (Laurence, 2019). Of these, the chronically absent (students who skip more than 15\%+ of school days in a year) are considered at-risk. Occasional absentees are at risk for becoming chronic absentees and our goal was to identify the subset of occasional absentees who may be classified as strategically absent and prevent them from becoming chronically absent.

Several strategies have been tried, with mixed results, in improving attendance: these have mostly been extrinsic in nature, directed at either rewarding attendance or punishing absences; some have looked at indirect efforts in influencing behavior usually by running campaigns to underline the link between attendance and higher grades, in hopes of improving attendance. The experiment did not make explicit claims; the suggestions were in the implications the students made themselves (Moore, 2004). However, such methods require a third party to impose the suggestion (which might be referred to as direct nudging), when it might be preferable to instill a sense of independence and self-motivation in students by having them make their own decision and selfsuggest their attendance. Teenagers in high school settings are famously rebellious, and our goal was to try and see if we could use intrinsic motivation to nudge behaviors, especially as open campuses become more of a staple in both secondary schools and colleges. Our efforts were aimed at understanding how enabling student choice in thinking about and making their own decisions could be used within behavioral frameworks to result in globally beneficial outcomes. The question we looked at was whether in a given context, priming and environmental nudges could influence attendance rates compared to baseline models using more direct persuasion methods.

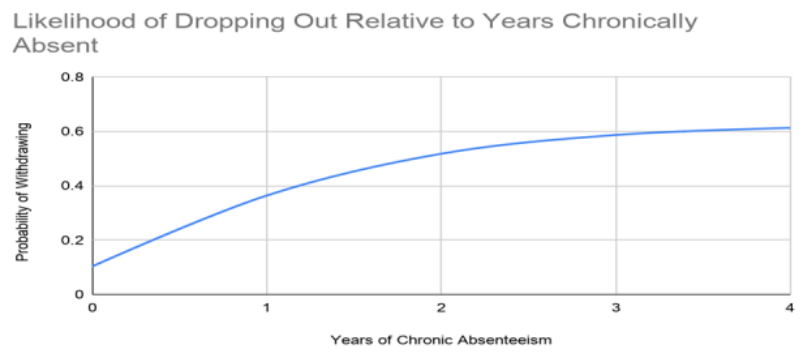


Fig.1 Utah dropout rates plotted against years with absenteeism rates of $15 \%$ or greater (Univ. of Utah, 2019)

\section{Related Work}

\section{Subliminal Influencing and Nudging}

In this work, our goal was to present students with information that might influence their decisions: to see if we could improve attendance through nudges. A nudge is an indirect suggestion that neither alters the choices available, nor the ability to decide upon an option, that promotes certain behaviors and outcomes (Thaler and Sunstein, 2008). Nudges have become a popular tool in the social engineering toolbox because of their effectiveness and ease of use in designing consistent experimental frameworks.

The primary framework that nudge and decision theorists agree upon usually employs two systems working in tandem, commonly referred to as System 1 and System 2 (Lin, Osman, Ashcroft, 2017). System 1 is the cognitive scheme that motivates our intrinsic, instinctual, autonomic responses: these tend to maintain biases and intuitions but are extremely reactive and highly consistent. System 1 responses often appear to be using minimal information and result in seemingly ad hoc judgements. System 2 tends to be System 1 's antithetical counterpart: the conscious part of reasoning, analytical and adaptive, but rather slow as well. For this reason, frameworks that are designed for nudging experiments frequently implement System 1 nudges to provoke biases and past associations in order to trigger a desired response.

However, we still know very little about how the two processes interact: we do not even know whether they act in parallel or serial fashion, whether they supplement each other or counteract, and when. The only thing clear is that neither model on its own can completely explain user behavior. Though there have been a number of experiments on using nudges to promote certain behaviors, the use of nudges has been controversial, since it brings up ethical issues regarding the manipulation of people: getting them to act, potentially against their will, is problematic, especially because of difficulty in monitoring (Hill, 2018). Although the utilization of nudges does not restrict any of the original options from being chosen -- sometimes described as "liberal paternalism," (Sunstein and Thaler, 2008), the ethical issues in implementing nudges are still heavily debated. Our experiment centers around using nudges to subconsciously incentivize students to act upon the systemic goal of attending class.

\section{Priming}

Priming is a specific form of persuasion that can be done via the environment. Priming is the process of seeding an idea into someone's implicit memory (System 1 frameworks) and exploiting that seed by calling upon it when needed. To prime one's thoughts is to temporarily restructure one's style of thinking. Generally, when someone imitates another's behavior, or is generally complimented, they tend to be more open to future criticism by that person, as they see the critiques in good faith, rather than as needless interference. The basis of priming is the use of the affective tone in an effort to restructure how the subject views the situation following the stimulus (Carlson, Charlin, and Miller, 1988). Priming functions as a cue that temporarily increases the likelihood of a desired outcome. It is important to keep in mind that the experiment should present an unrelated idea in a tone or form that will resonate strongly later; this provides a higher probability of unconscious behavior modification.

Among the most common implementations is to present something already in agreement with the subject's consciousness, to make them more likely to agree with future proposals. Priming can be used as a precursor to nudges; they reinforce future nudges before they even happen. One of the most ubiquitous priming ideologies is the idea of defaults; the predetermined, suggested option in the case the subject is indifferent or has no strong stance on the manner. In Austria, for instance, the organ donation policy is based upon opting out, leading to $90 \%$ donating; in the United States, the program is opt-in, leading to a mere $15 \%$ participation (Scheiber, 2012; Davidai, et al, 2012). These priming suggestions need not be directed towards a specific value or set of choices that the target already holds but can be used to prime people with the implication that the default was selected by an expert, adding a sense of credibility that justifies their selection. Priming and defaults are especially important for actions considered risky, or with unknown, costly outcomes (such as elections). It has been shown that risk is very hard to properly gauge, and people, in order to reduce the cognitive stress of decision making, revert instinctively to System 1 and rely on a set of predetermined approaches and biases (Tversky and Kahneman, 1974). Consequently, if high school students can be primed to more readily consider the need to attend classes, then they're more likely to do so. This was seen in Moore's experiment with showing the class his data: the students were primed to be more accepting of the correlation shown in the graph, which consequent results. This study incorporates priming in a manner that does not require either teacher and or authority intervention.

\section{Reactance: Inherent Rebellion}

Traditional models of persuasion would suggest that professional opinion is received positively and usually followed. This is what happens when people hear from doctors, lawyers and accountants. Authoritative recommendations both decrease the cognitive effort needed to make a choice and simultaneously increasing the confidence in it. However, under certain conditions, the opposite is observed: a decrease in satisfaction when presented with the choices, and the subsequent selection of a non-recommended option. This phenomenon is known as reactance: the backlash one experiences when a 
recommendation conflicts with other, previously established preferences; the cognitive dissonance from expected recommendations conflicting with the actual recommendation (Fitzsimons and Lehmann, 2004). For example, if a patient comes in to see a doctor convinced that they are ill, only to be told by the doctor that they are fine and need no treatment, the patient is more likely to feel the need to self-diagnose. This conflict in ideas usually leads to an even greater interest in the person's original preference and general discounting of the expert's recommendation. Reactance is especially prevalent in teenagers: conflicts between school administrators' exhortations and student behavior is ubiquitous. Our goal is to not only be aware of reactance as an issue but also make use of it: it can be exploited to specifically reduce the default option's presence; we will use a subset of nudges known as inverted nudges, where instead of encouraging a particular choice, they are used to diminish others (Spizman, 2018). We hope to subvert System 1 with targeted nudges of the unconscious so as to avoid potential reactance.

\section{Existing Studies}

Existing studies have already been conducted on improving attendance rates at institutions (Moore, 2004): as a teacher, Moore would show students in the class a graph that correlated high attendance with high academic performance, without explaining the implications of the graph; he left the interpretation of the graph to the students. His class attendance statistics improved. Others have shown that implementing policies that directly punish students for absenteeism is more effective in lieu of rewarding attendance as well (Self, 2012). While these studies present interesting ideas, they use direct intervention methods and are similar to well-known ideas in behavioral economics such as loss aversion. Our focus is on background efforts so as to avoid reactance that may inhibit following the suggestion; our goal is understand interventions and outcomes. Also, as mentioned earlier, all previous work on absenteeism was conducted in school districts that were more at risk. In those settings, student absenteeism was negatively related to academic self-perception, attitudes towards teacher and school, goal valuation, motivation/self-regulation, and academic performance along with many other non-scholastic factors (Balkis, Arslan, and Duru, 2016). Our study is interesting precisely for its complementary demographic: our school district is a high performing school district that should have very different underlying behavioral vectors.

\section{Experimental Setup}

Our experiments were conducted in one of the two high schools in the school district. Although absenteeism would not rank as a most prominent problem for school administrators in this district (competitive burn-out and unsustainably high goals probably rank higher), what makes our case interesting is that it has a surprisingly high rate of chronic absenteeism relative to competitiveness (US Dept of Education, 2016). In fact, the report strongly suggests that the absentees are of a more specific variant: those deemed as strategic absentees (Laurence, 2019). These strategic absentees aren't skipping classes because they don't want to study or because they are forced by involuntary economic conditions, but rather because they see it as a an optimization choice: they skip classes so that they can study or prepare for another class's commitment (i.e. test, quiz, presentation, etc.), whose importance is considered to be greater than the one they are skipping. Strategic absentees are skipping class not because they do not care for school, but rather because they do. (Consider this the school version of the First World Problem). Our goal is to convince students to value all classes, not just a select few that happen to think matter more. The 2017-2018 California Healthy Kids Survey found that, in a representative school district in Northern California, 7\% of 9th graders and $11 \%$ of 11 th graders cut classes regularly to prepare for another class. Strategic absentees are especially worth attempting to persuade, as many are certainly still elsewhere on campus, usually to leverage resources such as the library or student tutors.

Strategic absentees are characterized by their intermittent absences in one class coupled with their corresponding presence elsewhere on campus: thus, our interventions are expected to more likely to touch strategic absentees than chronic ones who may not be on campus at all. As we pointed out earlier, this distinguishes our study from other absenteeism work, in other parts of the nation, where the reasons for delinquency are different -- for instance, the student in question may be required to work since they need to augment their family's income. Even if the experimental results are true only for the demographic of academically high performing students driven to distraction by a competitive environment, we expect that the methodology and the results will still provide a means of discovering and using a district's primary motivators to incentivize attendance.

\section{Research Methodologies}

As stated, this study does not look to target all chronically absent students (not being on campus prevents us from reaching them with our interventions), but rather the strategically absent. Using the Moore (2004) model, we examined 4 variations of intervening suggestions relative to a baseline control. The classes used for our intervention settings were social studies classes: two US History classes (junior year) and three US Government classes (sophomore year), ranging in student population between 22 and 32 per class. The important detail across these wide variations in each class is that they are under the instruction of the same teacher, in the same classroom; the almost identical environments presented allow for minimal variance in the influence the class has on a given student (i.e., whether or not 


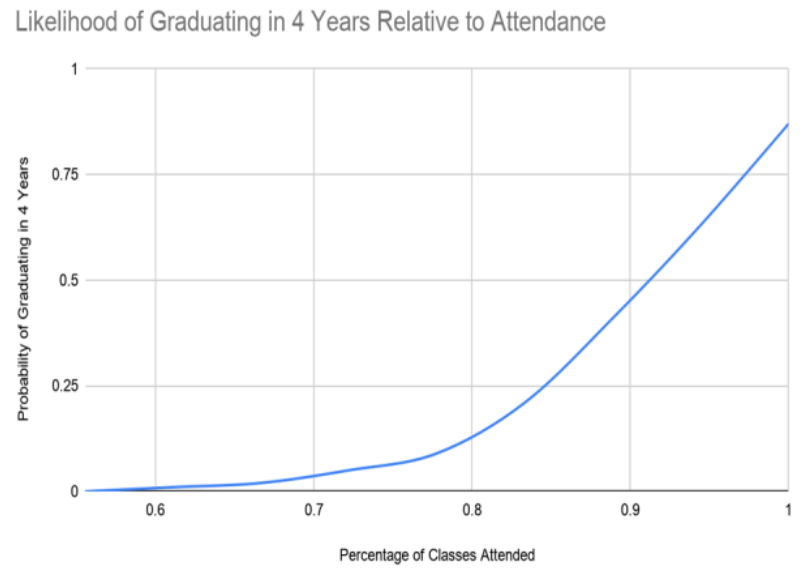

Figure 3a: Graph adapted from University of Chicago's E. Allensworth (2013) study to convey positive correlation between student attendance and likelihood of graduating.
Likelihood of Graduating in 4 Years Relative to Days Absent

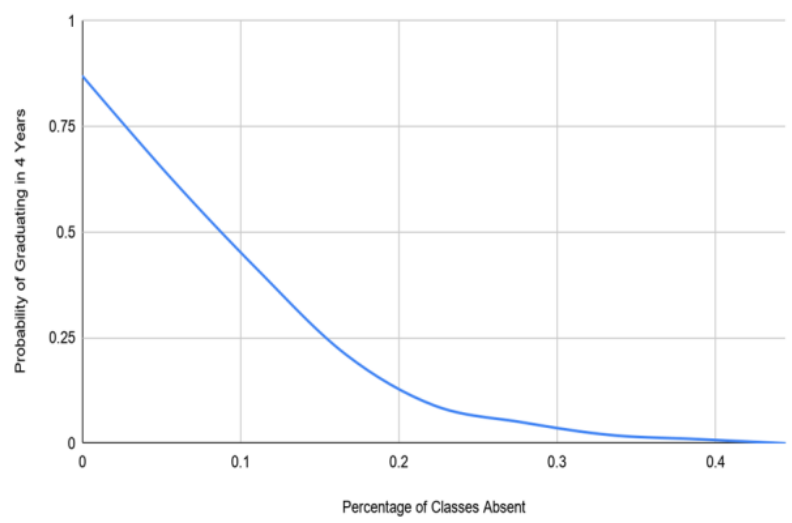

Figure 3b: Graph implied from University of Chicago's E. Allensworth (2013) study to convey negative correlation between student absence and unlikelihood of graduating. a student chooses to skip is dictated by how important/interesting the student views the class in place of another). Although the demographics between individual classes are almost certain to differ slightly, the fact that they are all exposed to the same curriculum with the same teacher in the same uniform school district makes for very similar environments; the goal is to observe relative changes in attendance records (percent observed), instead of the absolute changes (discrete count observed) of classes.

The first group (one classroom) was exposed to the original intervention Moore reported to be effective: the teacher discussed a correlation between high attendance and high performance in the classroom (Figure 3a). The control group was exposed to this direct intervention every alternate class period.

A second class had the same information presented to them, but indirectly: a poster was prominently placed in the classroom though there was no discussion led by the teacher on it. The poster appeared one day in the classroom and stayed there for the duration of the experiment. The students could view and try to understand the information independently.

Two other classes mirrored the scenarios above, but instead of implying a positive association with attendance, they provided a negative association with absenteeism (i.e., a graph correlating high absenteeism with low performance; see Figure 3b). This went on for three weeks.

These four variations were designed to measure the impact and influence of loss aversion, cognitive endowment, and reactance; similar to the effect of mood on persuasion, these scenarios sought to address the question of whether the manner of information presentation affects persuasion (i.e., is a positive connotation more effective at persuading than a negative one? Or vice versa?)

Data was collected using the teacher interface provided by Infinite Campus, in which official attendance is reported to the school's Student Information System (SIS), but only at aggregate levels. No individuals were identified, nor were any analyses done on any other data, such as academic performance. Strict anonymity controls were observed. The only absences and tardies collected were ones those that were unexcused: ones that were not waived by a parent for health, a pre-scheduled appointment, an emergency, or some other reason.

\section{Results}

The results of the five social studies classes' attendance records (Figure 4) exhibit distinct parallel trends between the four experimental models and the control, all showing similar peaks, troughs, and growth patterns across an observation period of three weeks. The classes are labeled according to which intervention was demonstrated and by whom (i.e., "+/teacher" refers to the class in which the positively correlating stimulus was demonstrated by the teacher). The graphs were constructed by taking the absolute value observed, divided by the number of students in the class, divided by class days observed. This gives us a dataset per class that can be compared to other classes using proportional data instead of absolute numbers, to compensate for slight differences in class sizes. The use of the line graph across classes is to be able to accurately relate the structure of each graph to the other, as similar structure between two or more 


\section{Summary of Experimental Results}

" = Tardies (Jan) - Absences (Jan) = - Tardies (Feb) - Absences (Feb) - C Chronic Absentee Rate

0.2

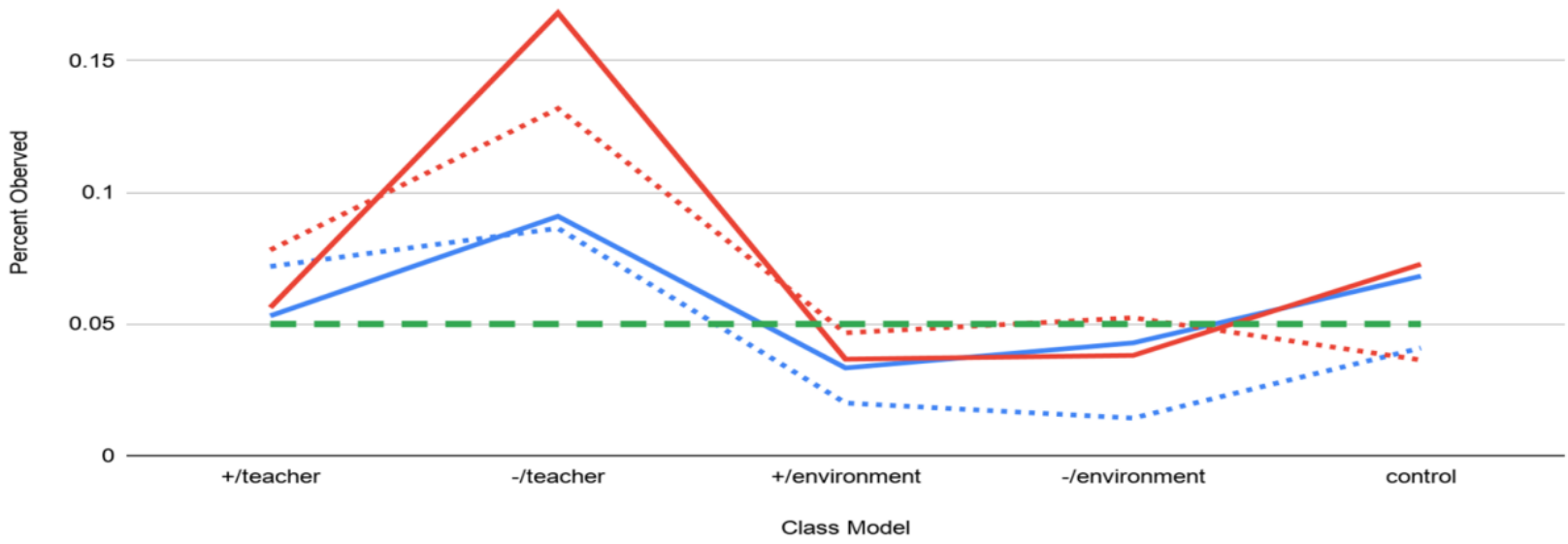

Figure 4: Observed data of recorded absences between 5 environmentally identical social studies classes as to isolate any extraneous factors impacting attendance and punctuality.

graphs indicate a similar impact across all classes. Using tardies as an indicator of punctuality, there appears to be a maintenance of graph structure and shape between January and February's tardies; however, the fact that this not reflected in the control indicates some broad overall, negative effect on punctuality. So, regardless of intervention style, the interventions resulted in a universal mild discouragement to attend. This corroborates with the presence of reactance in the student body, as introducing this experiment in the first place is viewed as a (mild) challenge to their attendance behaviors. More significant, however, is the absentee trend: although most observed classes are within the range of the expected $5.3 \%$ absentee rate, the class in which the teacher elaborated on Figure $3 b$ showed a substantial spike in absentees, with almost $17 \%$ of students skipping class across the three weeks.

\section{Conclusions}

The counter-intuitive finding appears to be that messaging did not have major impact, and the differences between the two (positive vs negative) were small. What is more surprising is that there was no substantial difference between the active teacher and the passive poster interventions in the classroom. Comparing all the classes, except "-/teacher", to the control, we observe that there was no significant decrease in absenteeism, but there was an across-the-board increase in tardies of approximately 4\%, which runs counter to the findings from Moore (2004). We were working with an already motivated classroom, so we did not expect large swings, and coupled with the fact that we targeted strategic rather than chronic absentees, our numbers may have been difficult to move dramatically. With a correlation coefficient $r=0.973197$ between January and February absences (excluding outliers), it can be argued that these experiments need to be conducted on much larger sample sets: perhaps with thousands rather than on dozens of students. However, the anomaly that did present itself is the "-/teacher" class seen in Figure 4: the class where the teacher used negative messaging to describe the effects of absenteeism, absences nearly doubled going up by $85 \%$. In this class, the teacher intervention increased the incidence of tardies by $4.5 \%$ and absenteeism by $7.7 \%$ : the difference between an additional student consistently tardy and an additional two that are consistently absent. If this were mapped to the entire school body, this would result in an additional 90 students who becoming chronically absent in a single school alone, and an additional 560 students across the entirety of the school district. This finding is particularly troubling because it suggests that the effect would be exacerbated in settings where the absenteeism rates were higher. It is as though the negative messaging amplified the reactance the students felt when their actions were critiqued, which supports the idea that negativity bias can amplify reactance; it's as if System 1 (negativity bias) working in tandem with System 2 (reactance). This amplification of reactance may have turned occasional absentees into regular ones, or tardies into absentees. The suggestion that they are being targeted for behaving "incorrectly," may cause them to find a justification for being late to this class specifically ('I need to go study and prepare for another class'). 


\section{Implications and Next Steps}

The primary takeaway from the results is that (i) fear is more effective at behavior modification - by a factor of two in our findings, and (ii) reactance can further amplify these effects. Our findings also illustrate that for significant change to occur, targeting has to be extremely specific so that students can apply the message to their individual selves; if students are encouraged to do something new, it is not as effective (working with defaults — attending class — is much easier). Reactance applies when the students know about the intervention and are aware of the intent behind it. For school administrations, these results could be useful for planning an effective messaging strategy.

It may be worth considering how reactance to different aspects of the message might counter/support each other. For example, the message "absenteeism is bad" resulted in reactance and a likely digging in of their heels on the part of the students. However, it may be worth investigating how to deflect the target of reactance from the goal (absenteeism) to something else: for instance, if the information were to be presented as "the school principal wants you to change your tardy and absentee habits", it is possible that the results could change, if the students react more strongly to the authority component in the message rather than absenteeism. This is worth testing to see if reactance order may impact the decision-making process.

The data, though small in sample size, also contributes to the field of study re-opening a popular question (attendance) in the context of two new aspects: motivated students driven not by a failure of fear but by the desire to succeed, and reactance as a parameter in their responses. Another caveat is that effects seen in a humanities class (history) may be different in other classes as underlying motivations may be different. Referring back to the idea of concurrent cognitive processes (such as loss aversion impacting reactance), an interesting question for future work is whether (and how) other cognitive effects might be combined.

Finally, the conclusion we wish to acknowledge is that persuasion is extraordinarily difficult. To predict exactly how messaging will impact a recipient, we can use our understanding of how theories of cognition will work, but we likely will never truly know the set of beliefs a user holds dear. However, if schools intend to address other issues beyond attendance, such as the rising vaping epidemic or the issue of cheating, knowing how their messaging is likely to be received by the student population is crucial to see real change.

Acknowledgments: I thank my Advanced Authentic Research (AAR) mentor and teachers who helped me work on this topic. This work would not have been possible without the support of the school administrators.

\section{References}

Balkis, M., Arslan, G., \& Duru, E. (2016). Absenteeism among high school students: Contributing factors. Educational Sciences: Theory and Practice, 16(6), 1819-1831. https://doi.org/10.12738/estp.2016.6.0125

Carlson, M., Charlin, V., \& Miller, N. (1988). Positive mood and helping behavior: A test of six hypotheses [PDF]. Journal of Personality and Social Psychology, 55(2), 211-229. https://doi.org/10.1037/0022-3514.55.2.211

Fitzsimons, G. J., \& Lehmann, D. R. (2004). Reactance to recommendations: When unsolicited advice yields contrary responses. Marketing Science, 23(1), 82-94. https://doi.org/10.1287

Heukelom, F. (2006). Kahneman and Tversky and the origin of behavioral economics. Tinbergen Institute Discussion Papers. Retrieved from SSRN database. (Accession No. 956887)

Hill, A. (2018). Why nudges coerce: Experimental evidence on the architecture of regulation. Science and Engineering Ethics, 1279-1295. https://doi.org/10.1007/s11948-017-9944-9

Kremer, I., Mansour, Y., \& Perry, M. (2014). Implementing the "wisdom of the crowd". Journal of Political Economics, 122(5), 988-1012. https://doi.org/10.1086/676597

Lin, Y., Osman, M., \& Ashcroft, R. (2017). Nudge: Concept, effectiveness, ethics. Basic and Applied Social Psychology, 39(6),

293-306. https://doi.org/10.1080/01973533.2017.1356304

Moore, R. (2004). Does improving developmental education students' understanding of the importance of class attendance improve students' attendance and academic performance? Research and Teaching in Developmental Education, 20(2). Retrieved from JSTOR database.

Scheiber, F. (2012) https://sparq.stanford.edu/solutions/opt-outpolicies-increase-organ-donation.

Davidai, S., Gilovich, T., \& Ross, L. (2012). The meaning of

default options for potential organ donors. Proceedings of the

National Academy of Sciences, 15201-15205.

Reid, L., \& Ellsworth-Krebs, K. (2019). Nudge(ography) and practice theories: Contemporary sites of behavioural science and poststructuralist approaches in geography? Progress in Human Geography, 43, 295-313. https://doi.org/10.1177/0309132517750773

Self, S. (2012). Studying absenteeism in principles of macroeconomics: Do attendance policies make a difference? The Journal of Economic Education, 43(3), 223-234. https://doi.org/10.1080/00220485.2012.686382

Spizman, L. M. (2018). The inverted nudge: An application of behavioral economic concepts to settlement outcomes. Journal of Legal Economics, 95-106. Retrieved from EBSCOhost database.

Thaler, R. H., \& Sunstein, C. R. (2008). Nudge: Improving decisions about health, wealth, and happiness. New Haven, Conn.: Yale University Press.

Tversky, A., \& Kahneman, D. (1974). Judgment under uncertainty: Heuristics and biases. Science, 185(4157), 11241131. doi.org/10.1126/science.185.4157.1124

United States Government Department of Education. (2019, January). Chronic absenteeism in nation's schools. Retrieved from Department of Education website: https://www2.ed.gov/datastory/chronicabsenteeism.html 